\title{
La posición supina de Valdivia como mejor opción para la cirugia percutánea de los cálculos renales en el paciente con obesidad mórbida
}

\author{
L.A. Fariña Pérez, E.R. Zungri Telo \\ Servicio de Urología. Hospital Povisa. Vigo. Pontevedra. \\ Actas Urol Esp 2005; 29 (10): 997-1000
}

\section{RESUMEN}

LA POSICIÓN SUPINA DE VALDIVIA COMO MEJOR OPCIÓN PARA LA CIRUGÍA PERCUTÁNEA DE LOS CÁLCULOS RENALES EN EL PACIENTE CON OBESIDAD MÓRBIDA

Introducción: El tratamiento del paciente obeso grave afecto de cálculos renales sintomáticos, supone un interesante reto urológico. El tratamiento mediante litotricia extracorpórea con frecuencia no es posible por diversas razones, y en algunos centros estos pacientes constituyen una de las indicaciones residuales de cirugía abierta de los cálculos.

Material y método: Dos mujeres con índice de masa corporal mayor de $50 \mathrm{~kg} / \mathrm{m}^{2}$, afectas de cálculos renales sintomáticos, fueron tratadas mediante nefrolitotomía percutánea bajo anestesia en la posición de Valdivia (decúbito supino ligeramente lateralizado). Se usaron los instrumentos estándar y el ureteroscopio semi-rígido, a través de una vaina de Amplatz proximalmente transfixiada con una sutura de polipropileno para evitar su pérdida bajo la piel. Ambas pacientes quedaron libres de cálculos sin morbilidad significativa.

Discusión: Las ventajas de la posición de Valdivia en este tipo de pacientes son claras, tanto para la comodidad del equipo quirúrgico, pues se puede prescindir de la ayuda y del tiempo necesario para voltear al paciente anestesiado a la posición de prono, como para el discurrir de la anestesia, pues son bien conocidas las restricciones cardio-respiratorias creadas por una anestesia general en prono, que son más graves en el paciente obeso. Esta posición nos parece más cómoda que otras recomendadas para la nefrolitotomía percutánea en el paciente obeso, como son el decúbito prono en una mesa de cirugía del raquis, o la posición de decúbito lateral, que puede comprometer el control radiológico de la intervención.

Palabras clave: Litiasis renal. Nefrolitotomía percutánea. Obesidad mórbida.

\section{ABSTRACT \\ VALDIVIA SUPINE POSITION AS THE BEST OPTION FOR PERCUTANEOUS SURGERY OF RENAL CALCULI IN MORBIDLY OBESE PATIENTS}

Introduction: Treatment of the morbidly obese patient with symptomatic renal calculi is an interesting urological challenge. Extracorporeal shock wave lithotripsy is frequently not possible for several reasons, and many urological centers match these patients as one of the residual indications for open surgery of kidney lithiasis.

Material and method: Two patients with body mass index more than $50 \mathrm{k} / \mathrm{m}^{2}$ and symptomatic kidney stones were treated with percutaneous nephrolithotomy in the supine position described by Valdivia (slightly lateralized supine decubitus). Standard instrumentation for percutaneous surgery and the semi-rigid ureteroscopy were used, through an Amplatz sheath proximally transfixed with a polypropylene stitch, so to avoid its lost under the fatty skin. Both patients were rendered stone-free without significant morbidity.

Discussion: The advantages of Valdivia position in those patients are clear, both for the commodity of the surgical team, -because time may be spared and help needed to mobilized the patient is less-, as well as for what concerns to the anesthesia, because cardio-respiratory restrictions created by a general anesthesia in prone are greater and more severe in the obese patient. This position seems to be more suitable than other ones recommended for percutaneous nephrolithotomy in the obese patients, such as prone decubitus on a surgical saddle, or lateral decubitus position, that may compromise the radiological control of the operation.

Keywords: Renal lithiasis. Percutaneous nephrolithotomy. Morbidly obese patient. 
E tratamiento del paciente obeso grave afecto de cálculos renales sintomáticos, supone un interesante reto urológico. Con frecuencia el tratamiento mediante litotricia extracorpórea por ondas de choque no es posible, ya sea porque los litotriptores no admiten el peso del paciente, o porque existe una excesiva distancia entre la piel y el foco litiásico, incompatible con las prestaciones del litotriptor. Por este motivo, en algunos centros, estos pacientes constituyen una de las indicaciones residuales de cirugía abierta de los cálculos $^{1,2}$, lo que supone un riesgo añadido, por las potenciales importantes complicaciones anestésicas y de la incisión quirúrgica a las que están expuestos. Presentamos nuestra experiencia reciente con la posición descrita por Valdivia et al., de decúbito supino ligeramente lateralizado ${ }^{3}$, para el tratamiento mediante nefrolitotomía percutánea bajo anestesia, del paciente obeso grave afecto de cálculos renales sintomáticos.

\section{PACIENTES Y MÉTODO}

En el último año hemos tratado en la posición supina de Valdivia (decúbito supino ligeramente lateralizado) a dos mujeres con índice de masa corporal mayor de $50 \mathrm{~kg} / \mathrm{m}^{2}$, estando una de ellas en espera de cirugia bariátrica. Ambas estaban afectas de cálculos sintomáticos: de cáliz inferior y unión pielo-ureteral en un caso, y de molde pielocalicial inferior en otro caso. Se usaron los instru-

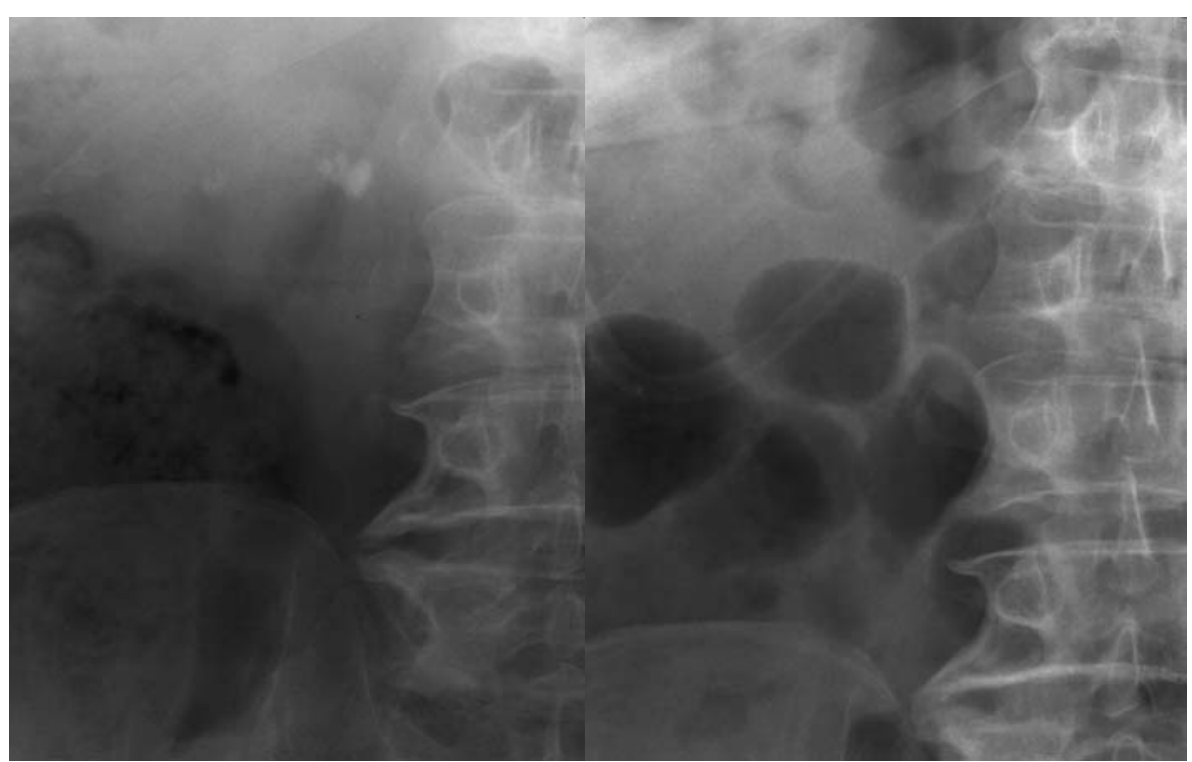

FIGURA 1. RX simple antes (izquierda) y después (derecha) de nefrolitotomia percutánea de cálculos radioopacos en la unión pielo-ureteral y cáliz inferior. mentos estándar (nefroscopio operatorio) y el ureteroscopio semi-rígido, a través de una vaina de Amplatz de $30 \mathrm{~F}$ proximalmente transfixiada con una sutura de polipropileno para evitar su pérdida bajo la piel. Ambas pacientes quedaron libres de cálculos sin morbilidad significativa.

Caso 1. Mujer de 50 años con índice de masa corporal de $52 \mathrm{~kg} / \mathrm{m}^{2}$, que presenta con síntomas agudos un cálculo de la unión pieloureteral derecha de 10 por $10 \mathrm{~mm}$, con leve ectasia renal, asociado a pequeños cálculos de cáliz inferior (Fig. 1). Se intentó hacer litotricia extracorpórea en un litotriptor Compact Delta (Dornier), que resultó imposible por la excesiva distancia piel-cálculo. Bajo anestesia general se realizó empuje retrógrado del cálculo a la pelvis renal, seguido de nefrolitotomía percutánea en posición supina de Valdivia. La vaina de Amplatz de tamaño estándar hubo de sujetarse con un punto de sutura para evitar que se perdiera bajo la piel. Se usó el nefroscopio para extraer con pinza microcálculos de cáliz inferior y el ureteroscopio semi-rígido para alcanzar la pelvis renal y fragmentar mediante litotricia neumática y extraer con pinza, el cálculo de mayor tamaño .

Caso 2. Mujer de 45 años con índice de masa corporal de $56 \mathrm{~kg} / \mathrm{m}^{2}$, con antecedente de ureteroscopia y litotricia intracorpórea por litiasis obstructiva de uréter lumbar izquierdo. Presenta ahora una gruesa litiasis pielocalicial izquierda sintomática, de gran volumen, no obstructiva y de baja densidad radiológica (Fig. 2), que se recomendó tratar antes de realizar una cirugía bariátrica que tenía programada. Se realizó nefrolitotomía percutánea bajo anestesia regional en posición de Valdivia (Fig. 3), con ayuda de una incisión de unos $4 \mathrm{~cm}$ de la piel y tejido graso, que dejó al descubierto el músculo del flanco y que se mantuvo abierta con un separador 


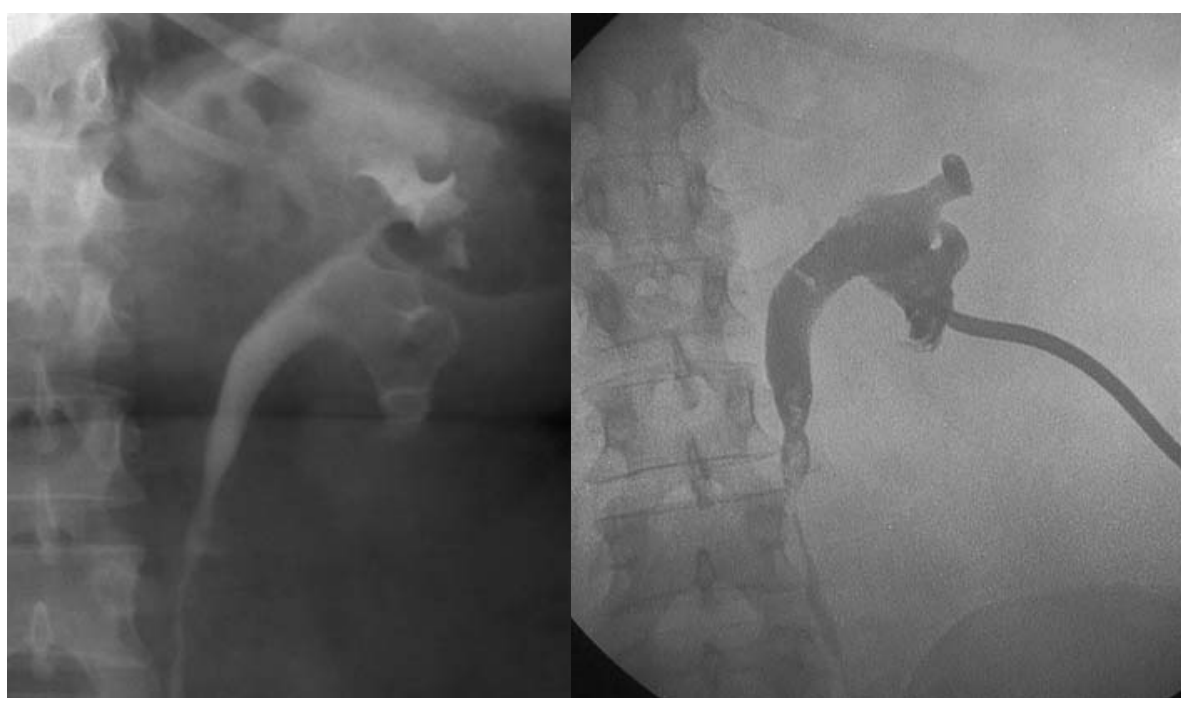

FIGURA 2. Urografia (izquierda) y pielografia por nefrostomia (derecha), antes y después de nefrolitotomía percutánea de cálculo úrico pielocalicial inferior izquierdo en riñón con buena función.

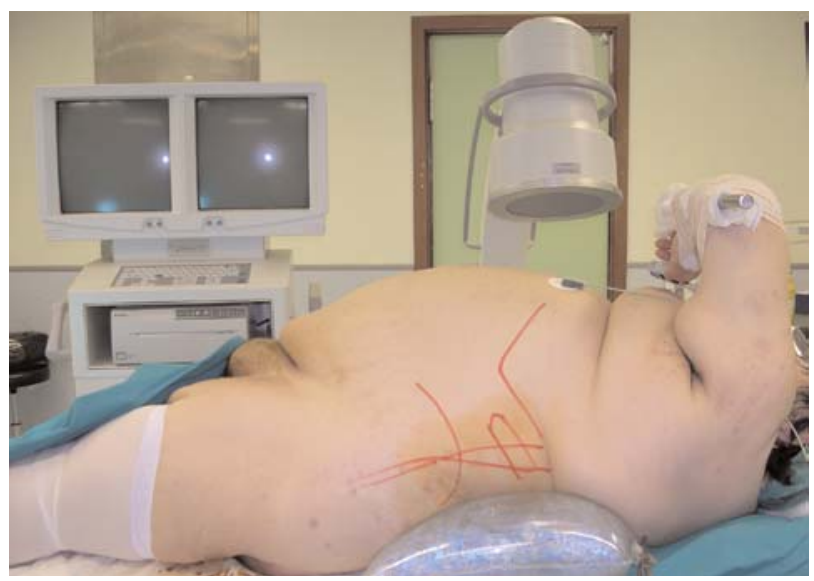

FIGURA 3. Mujer con indice de masa corporal $56 \mathrm{k} / \mathrm{m} 2$ en posición de Valdivia para nefrolitotomia percutánea, bajo anestesia regional.

autostático de Adson. Fue posible de este modo usar la vaina de Amplatz de tamaño estándar, asegurada externamente del modo señalado antes. Se usó el nefroscopio estándar y litotricia ultrasónica para evacuar el cálculo. En la pielografía postoperatoria por la nefrostomía se observaron algunos restos litiásicos en uréter distal, que fueron espontáneamente eliminados inmediatamente después de pinzar la nefrostomía.

\section{DISCUSION}

No hemos optado por la posición supina en todos los casos de nefrolitotomía percutánea, aunque es una opción que, después de la amplia experiencia comunicada por el grupo de Valdivia, tiene algunos partidarios en nuestro país ${ }^{4}$ y en otros ${ }^{5}$. La razón es que aún somos excépticos sobre si siempre será posible, en esta posición, realizar una buen acceso inicial en litiasis de difícil tratamiento, como es el caso de necesitar acceder a la vía urinaria a través de los cálices medio o superior, en caso de necesitar varios accesos, en caso de cálculo en divertículo calicial y en situaciones de parecida complejidad. Además, el amplio desplazamiento anteromedial del riñón que tiene lugar en esta posición nos parece una circunstancia desfavorable a la hora del acceso inicial a la vía urinaria.

Sin embargo, las enseñanzas de estos dos casos nos hacen pensar que las ventajas de la posición supina de Valdivia en los pacientes con obesidad mórbida son claras, tanto para la comodidad del equipo quirúrgico, -pues se puede prescindir de la ayuda y del tiempo necesario para voltear al paciente anestesiado a la posición de prono-, como para el discurrir de la anestesia, pues son bien conocidas las restricciones cardiorespiratorias creadas por una anestesia general en prono, que son más graves en el paciente obeso. Esta posición nos parece en principio más cómoda que otras recomendadas para la nefrolitotomía percutánea en el paciente obeso, como son el decúbito prono en una mesa de cirugía del raquis $^{6}$, o la posición de decúbito lateral, que puede comprometer el control radiológico de la intervención ${ }^{7}$.

Los obesos son un grupo de pacientes que pueden potencialmente beneficiarse de las nuevas indicaciones de tratamiento mediante ureteroscopia retrógrada y litotricia intracorpórea de los cálculos intrarrenales ${ }^{8,9}$, actualmente limitadas por la dificultad de la fragmentación y de la evacuación de fragmentos múltiples mediante instrumentos de tan reducido calibre. En el futuro, tal vez esta técnica menos invasiva se depure y sea 
más eficiente y menos costosa. Entretanto, la nefrolitotomía percutánea en posición de Valdivia puede hacer menores las dificultades que el tratamiento de la litiasis urinaria todavía tiene para los pacientes con obesidad grave.

\section{REFERENCIAS}

1. Pascual X, Ortiz JC, Baxarias P. Obesidad y anestesia. Act Fund Puigvert 2003;22:138-140.

2. Paik ML, Wainstein MA, Spirnak JP, Hampel N, Resnick MI. Current indications for open stone surgery in the treatment of renal and ureteral calculi. J Urol 1998;159:374-378.

3. Valdivia JG, Valle J, López JA, Villarroya S, Ambroj C, Ramírez M, et al. Technique and complications of percutaneous nephroscopy: experience with 557 patients in the supine position. J Urol 1998;160:1975-1978.

4. Gallego JA, Gamarra M, Camargo I, Jorge A, Gurtubay I, Bildasola A, et al. Acceso endourológico integral a la vía urinaria. Video Arch Esp Urol 2004;16(2),vídeo 6.

5. Shoma AM, Eraky I, El-Kenawy MR, El-Kappany HA. Percutaneous nephrolithotomy in the supine position: technical aspects and functional outcome compared with the prone technique. Urology 2002;60:388-392.
6. Turner CD, Anderson J, Gerber GS. Improved patient positioning for percutaneous nephroscopic procedures using a surgical saddle. J Urol 2000;163:199-200.

7. Gofrit ON, Shapiro A, Donchin Y, Bloom AI, Shenfeld OZ, Landau $\mathrm{EH}$, et al. Lateral decubitus position for percutaneous nephrolithotripsy in the morbidly obese or kyphotic patient. J Endourol 2002;16:383-386 .

8. Andreoni C, Afane J, Olweny E, Clayman RV. Flexible ureteroscopic lithotripsy: first-line therapy for proximal ureteral and renal calculi in the morbidly obese and superobese patient. J Endourol 2001;15:493-498.

9. Dash A, Schuster TG, Hollenbeck BK, Faerber GJ, Wolf JS Jr. Ureteroscopic treatment of renal calculi in morbidly obese patients: a stone-matched comparison. Urology 2002;60:393-397.

Dr. L.A. Fariña Pérez

Servicio de Urología. Hospital POVISA

Salamanca 5

36211 Vigo (Pontevedra)

E-mail: luisfarina@yahoo.com

(Trabajo recibido el 13 de abril 2005) 